\title{
The Average-Case Time Complexity of Certifying the Restricted Isometry Property
}

\author{
Yunzi Ding*1, Dmitriy Kunisky ${ }^{\ddagger 1}$, Alexander S. Wein ${ }^{\S 1}$, and Afonso S. Bandeira ${ }^{\llbracket 2}$ \\ ${ }^{1}$ Department of Mathematics, Courant Institute of Mathematical Sciences, New York \\ University, USA \\ ${ }^{2}$ Department of Mathematics, ETH Zurich, Switzerland
}

\begin{abstract}
In compressed sensing, the restricted isometry property (RIP) on $M \times N$ sensing matrices (where $M<N)$ guarantees efficient reconstruction of sparse vectors. A matrix has the $(s, \delta)$ RIP property if behaves as a $\delta$-approximate isometry on $s$-sparse vectors. It is well known that an $M \times N$ matrix with i.i.d. $\mathcal{N}(0,1 / M)$ entries is $(s, \delta)$-RIP with high probability as long as $s \lesssim \delta^{2} M / \log N$. On the other hand, most prior works aiming to deterministically construct $(s, \delta)$-RIP matrices have failed when $s \gg \sqrt{M}$. An alternative way to find an RIP matrix could be to draw a random gaussian matrix and certify that it is indeed RIP. However, there is evidence that this certification task is computationally hard when $s \gg \sqrt{M}$, both in the worst case and the average case.

In this paper, we investigate the exact average-case time complexity of certifying the RIP property for $M \times N$ matrices with i.i.d. $\mathcal{N}(0,1 / M)$ entries, in the "possible but hard" regime $\sqrt{M} \ll s \lesssim M / \log N$. Based on analysis of the low-degree likelihood ratio, we give rigorous evidence that subexponential runtime $N^{\tilde{\Omega}\left(s^{2} / M\right)}$ is required, demonstrating a smooth tradeoff between the maximum tolerated sparsity and the required computational power. This lower bound is essentially tight, matching the runtime of an existing algorithm due to Koiran and Zouzias KZ14. Our hardness result allows $\delta$ to take any constant value in $(0,1)$, which captures the relevant regime for compressed sensing. This improves upon the existing average-case hardness result of Wang, Berthet, and Plan [WBP16], which is limited to $\delta=o(1)$.
\end{abstract}

\footnotetext{
*Email: yding@nyu.edu. Partially supported by NSF grant DMS-1712730.

${ }^{\ddagger}$ Email: kunisky@cims.nyu.edu. Partially supported by NSF grants DMS-1712730 and DMS-1719545.

${ }^{\S}$ Email: awein@cims.nyu.edu. Partially supported by NSF grant DMS-1712730 and by the Simons Collaboration on Algorithms and Geometry.

`Email: bandeira@math.ethz.ch. Part of this work was done while ASB was with the Department of Mathematics at the Courant Institute of Mathematical Sciences, and the Center for Data Science, at New York University; and partially supported by NSF grants DMS-1712730 and DMS-1719545, and by a grant from the Sloan Foundation.
} 


\section{Introduction}

\subsection{Restricted Isometry Property}

For measuring and reconstructing high-dimensional sparse signals, the compressed sensing technique introduced by Candès and Tao [CT05 and Donoho Don06 has demonstrated state-of-the-art efficiency and effectiveness in theory and practice. A central property on the sensing matrix, known as the restricted isometry property (RIP) Can08, requires that the matrix approximately preserves the norm of sparse vectors.

Definition 1.1. A matrix $A \in \mathbb{R}^{M \times N}$ is said to satisfy the $(s, \delta)$-RIP if

$$
(1-\delta)\|x\|^{2} \leq\|A x\|^{2} \leq(1+\delta)\|x\|^{2}
$$

for any $x \in \mathbb{R}^{N}$ with $\|x\|_{0} \leq s$. Here $\|\cdot\|$ denotes the vector $\ell^{2}$ norm, and $\|\cdot\|_{0}$ denotes the number of nonzero entries of a vector.

In the context of compressed sensing, in pursuit of reducing the dimension, we are interested in the case $M<N$. An $(s, \delta)$-RIP sensing matrix with $\delta<\sqrt{2}-1$ allows for efficient reconstruction of an $s / 2$-sparse $N$-dimensional signal from $M$ linear measurements in the compressed sensing framework Can08, CZ13a, CZ13b, FR13. From a practical point of view, given desired parameters $M, N, s, \delta$, one would like to construct an $(s, \delta)$-RIP matrix suitable for use as a sensing matrix. It appears to be very difficult to deterministically construct RIP matrices for $s \gg \sqrt{M}$, a phenomenon known as the "square bottleneck" Mix15, BFMM16, BMM17, Gam18; in fact, the only known success is due to $\left[\mathrm{BDF}^{+} 11\right]$, which constructed $(s, \delta)$-RIP matrices for $s \geq M^{1 / 2+\epsilon}$ for a small constant $\epsilon>0$. Readers may refer to BFMW13 for more details.

Meanwhile, randomized algorithms have seen much success in breaking the "square bottleneck" CT05, BDDW08, FR13. For example, by simply sampling an $M \times N$ matrix with i.i.d. $\mathcal{N}(0,1 / M)$ entries, one obtains a $(s, \delta)$-RIP matrix with high probability (i.e., probability $1-o(1)$ ), so long as $s \lesssim \delta^{2} M / \log N$. While such randomized algorithms generate RIP matrices with more desireable parameters $s, \delta$ than the known deterministic constructions, they suffer from a potential drawback: it is not guaranteed that the output is always $(s, \delta)$-RIP as desired. This motivates the following task known as RIP certification, in which we discard non-RIP samples and confidently keep a sample only when it is indeed RIP.

Problem 1.2 (RIP certification). Given a matrix $A \in \mathbb{R}^{M \times N}$, a positive integer $s$, and $\delta \in(0,1)$, output either "yes" or "no" according to the following rules. If $A$ is not $(s, \delta)$-RIP, the output must always be "no". If $A$ has i.i.d. $\mathcal{N}(0,1 / M)$ entries, the output must be "yes" with high probability.

Note that this allows false negative errors but not false positive errors, so that such a certifying procedure allows us to reliably obtain an RIP matrix by sampling random matrices until the certifier outputs "yes".

The worst-case problem of deciding (with certainty) whether or not a given matrix is $(s, \delta)$ RIP is NP-hard [TP13, BDMS13, even if the input is guaranteed to either be RIP or far from RIP [Wee17] (see also [KZ14, NW14] which require stronger assumptions than $P \neq N P$ ). All known algorithms for this worst-case task DeV07, AHSC09, FMT12, BFMW13, require time $N^{\tilde{\Omega}(s)}$, which is the time required to enumerate all possible support sets $S \subseteq[N]$ of cardinality $s$.

For the average-case RIP certification problem (Problem 1.2), it is shown in [WBP16] that thresholding $\left\|A^{\top} A-I_{N}\right\|_{\infty}$ gives a polynomial-time certifier of $(s, \delta)$-RIP for i.i.d. sub-gaussian matrices in the regime $s \lesssim \delta \sqrt{M / \log N}$. Note, however, that this does not help surpass the "square 
bottleneck". In fact, the same work [WBP16] shows that for any $\epsilon>0$, when $s \geq\left(\delta^{2} M / \log N\right)^{1 / 2+\epsilon}$, no polynomial-time certifier exists for the average-case task, conditional on an assumption about detecting dense subgraphs, which is a weaker assumption than the planted clique hypothesis (i.e., their assumption is implied by the planted clique hypothesis). However, this result only shows hardness in the regime $\delta=o(1)$.

\subsection{Our Contributions}

In this paper, we further investigate the average-case hardness of Problem 1.2, For any fixed $\delta \in(0,1)$, in the "possible but hard" regime $\sqrt{M} \ll s \lesssim M / \log N$, we give evidence that $(s, \delta)$ RIP certification requires time $N^{\tilde{\Omega}\left(s^{2} / M\right)}$ with an analysis of the low-degree likelihood ratio (see Section 1.4). Our lower bound is optimal, as it matches the runtime of the lazy algorithm proposed in [KZ14]. Here and throughout, the notation $\tilde{\Omega}, \tilde{O}$, and $\tilde{\Theta}$ hide factors of $\log N$, while $\lesssim$ hides a constant factor.

The strategy for our lower bound is to give a reduction to Problem 1.2 from a certain hypothesis testing problem in the negatively-spiked Wishart model. Our arguments will be based on two distributions over $\mathbb{R}^{M \times N}$, namely the distribution $\mathbb{Q}$ of i.i.d. Gaussian matrices and a distribution $\mathbb{P}$ over matrices which have a sparse vector planted in their null-space. We start with a proof that, in the regime $\sqrt{M} \ll s \lesssim M / \log N, A \sim \mathbb{Q}$ is RIP with high probability while $A \sim \mathbb{P}$ is non-RIP with high probability. We then argue that it is computationally hard to distinguish $\mathbb{P}$ from $\mathbb{Q}$, assuming the low-degree conjecture (see Section 1.4); in other words, we prove that the class of low-degree polynomial algorithms requires time $N^{\tilde{\Omega}\left(s^{2} / M\right)}$ to distinguish $\mathbb{P}$ from $\mathbb{Q}$. From this we infer the hardness of RIP certification.

For the matching upper bound, let us give a brief overview of the lazy algorithm in [KZ14. The main step of the algorithm involves exhaustive search over subsets of $[N]$ with cardinality $r \approx s^{2} / M$, which are interpreted as the possible supports of $r$-sparse vectors in $\mathbb{R}^{N}$. As $s$ ranges from $\sqrt{M}$ up to $M / \log N$, the runtime of the algorithm $\left(N^{\tilde{O}\left(s^{2} / M\right)}\right)$ smoothly interpolates between polynomial $\left(N^{O(1)}\right)$ and exponential $\left(N^{\tilde{O}(M)}\right)$. Our lower bound suggests that this is in fact the optimal tradeoff between runtime and sparsity.

Our main contribution in this paper is a precise understanding of the computational power needed for the average-case certification of $(s, \delta)$-RIP, namely $N^{\tilde{\Theta}\left(s^{2} / M\right)}$. In contrast, the previous average-case hardness result of [WBP16] only suggests that at least $N^{\log (N)}$ time is required, since (like planted clique) the dense subgraph problem that they give a reduction from can be solved in time $N^{\log (N)}$. Another strength of our lower bound is that it applies for any fixed $\delta \in(0,1)$, whereas the hardness result of [WBP16] is restricted to $\delta=o(1)$. In other words, we are showing that even an easier certification problem is hard. Since any fixed $\delta<\sqrt{2}-1$ is sufficient for compressed sensing applications, our result is the first average-case lower bound to capture the entire regime of relevant $\delta$ values.

\subsection{Spiked Wishart Model and Hardness of RIP Certification}

Definition 1.1 tells us that a matrix is not $(s, \delta)$-RIP for any $\delta \in(0,1)$ if there exists an $s$-sparse vector in its kernel. The computational hardness of RIP certification in this paper is based on the following fact:

Any certifier for $(s, \delta)$-RIP (a solution to Problem 1.2) can be used to distinguish a random matrix from any matrix that has an $s$-sparse vector in its kernel. 
Note that the operation on $A \in \mathbb{R}^{M \times N}$ that projects each row of $A$ onto the subspace $\{x\}^{\perp}$ for some $s$-sparse vector $x \in \mathbb{R}^{N}$ adds $x$ to the kernel of the resulting matrix, and hence deprives the matrix $A$ of its $(s, \delta)$-RIP property. Therefore, a certifier should be able to tell whether an $(s, \delta)$-RIP matrix $A$ has undergone this operation. We will make use of the classical spiked Wishart model, which captures the property of the row-wise projection.

Definition 1.3 (Spiked Wishart model). Let $\mathcal{X}=\left(\mathcal{X}_{N}\right)$ be a distribution over $\mathbb{R}^{N}$, and let $\beta \in$ $[-1,+\infty)$. We define two distributions over $\mathbb{R}^{M \times N}$, where $A \in \mathbb{R}^{M \times N}$ is taken as follows:

- Under $\mathbb{Q}=\mathbb{Q}_{N, M}$, draw each row $u_{i}^{\top}(i=1,2, \ldots, M)$ of $A$ i.i.d. from $\mathcal{N}(0, I)$.

- Under $\mathbb{P}=\mathbb{P}_{N, M, \beta, \mathcal{X}}$, draw $x \sim \mathcal{X}$. If $\beta\|x\|^{2} \geq-1$, then draw each row $u_{i}^{\top}(i=1,2, \ldots, M)$ of $A$ i.i.d. from $\mathcal{N}\left(0, I+\beta x x^{\top}\right)$; otherwise, draw each row $u_{i}^{\top}(i=1,2, \ldots, M)$ of $A$ i.i.d. from $\mathcal{N}(0, I)$.

We call $\mathbb{P}$ the planted model and $\mathbb{Q}$ the null model. We define the spiked Wishart model as the two distributions taken together: $(\mathbb{P}, \mathbb{Q})=\operatorname{Wishart}(N, M, \beta, \mathcal{X})$.

We will consider spike priors $\mathcal{X}$ normalized so that $\|x\| \approx 1$, ensuring that $\beta\|x\|^{2} \geq-1$ is a highprobability event under $\mathbb{P}$. The condition $\beta\|x\|^{2} \geq-1$ in the definition of $\mathbb{P}$ ensures that the covariance matrix $I+\beta x x^{\top}$ is positive semidefinite. Our case of interest will be $\beta<0$, in which case $\mathbb{P}$ can be viewed as the row-wise partial projection of $\mathbb{Q}$ onto $\{x\}^{\perp}$, where $x$ is taken from the prior distribution $\mathcal{X}$. In fact, for any $u \sim \mathcal{N}\left(0, I+\beta x x^{\top}\right)$ where $\|x\|=1$, we have

$$
\mathbb{E}\langle u, x\rangle^{2}=1+\beta<1=\mathbb{E}\langle u, y\rangle^{2}, \quad \text { for any unit-norm } y \perp x
$$

which tells us that the rows of $A$ under $\mathbb{P}$ have low correlation to the subspace $\{x\}$ but high correlation to the subspace $\{x\}^{\perp}$.

Since we are interested in the signal $x$ being sparse, we will take the spike prior $\mathcal{X}$ to be the following sparse Rademacher prior.

Definition 1.4 (Sparse Rademacher prior). Given $\rho \in(0,1)$, the sparse Rademacher prior $\mathcal{X}_{N}^{\rho}$ is the distribution over $\mathbb{R}^{N}$ where for $x \sim \mathcal{X}_{N}^{\rho}$ each entry $x_{i}$ is distributed independently as

$$
x_{i}=\left\{\begin{aligned}
\frac{1}{\sqrt{\rho N}} & \text { with probability } \frac{\rho}{2}, \\
-\frac{1}{\sqrt{\rho N}} & \text { with probability } \frac{\rho}{2}, \\
0 & \text { with probability } 1-\rho .
\end{aligned}\right.
$$

Note that $x \sim \mathcal{X}$ has $\mathbb{E}\|x\|^{2}=1$ and $\mathbb{E}\|x\|_{0}=\rho N$. In order for $x$ to be $s$-sparse with high probability, we will take $\rho=s /(2 N)$.

We now introduce the logic for demonstrating the average-case hardness of certifying RIP for gaussian random matrices. The following proposition serves as an outline of the proof of the lower bound. The informal assertions (1), (2) and (3) are made rigorous in Section 2, in Lemma 2.1, Theorem 2.2 and Theorem 2.3 respectively.

Proposition 1.5 (Informal). Fix a constant $\delta=(0,1)$ and let $\epsilon=\frac{1-\delta}{2(1+\delta)}$. Consider an asymptotic regime where $N \rightarrow \infty$ and where $M=M(N)$ and $s=s(N)$ scale with $N$ such that $M \leq N$, $M \rightarrow \infty$ and $s \rightarrow \infty$. Consider the sequence of spiked Wishart models with sparse Rademacher prior $\left\{\text { Wishart }\left(N, M,-(1-\epsilon), \mathcal{X}_{N}^{\rho}\right)\right\}_{N \in \mathbb{Z}^{+}}$where $\rho=s /(2 N)$. The following three properties hold in the limit $N \rightarrow \infty$. 
(1) For $A \sim \mathbb{P}, \frac{1}{\sqrt{M}} A$ is not $(s, \delta)$-RIP with high probability.

(2) For $A \sim \mathbb{Q}, \frac{1}{\sqrt{M}} A$ is $(s, \delta)$-RIP with high probability, provided $s \lesssim M / \log N$.

(3) Conditional on the low-degree conjecture (see Section 1.4), any algorithm to distinguish $\mathbb{P}$ from $\mathbb{Q}$ with error probability o(1) requires time $N^{\tilde{\Omega}\left(s^{2} / M\right)}$.

In light of assertions (1) and (2), any certifier for $(s, \delta)$-RIP (a solution to Problem 1.2) can be used to distinguish $\mathbb{P}$ from $\mathbb{Q}$ with error probability $o(1)$. Thus (3) implies that, conditional on the low-degree conjecture, any algorithm to solve Problem 1.2 requires time $N^{\tilde{\Omega}\left(s^{2} / M\right)}$.

The problem of distinguishing two sequences of distributions falls into the setting of hypothesis testing. In the following Section 1.4, we discuss a general method to predict computational hardness of such tasks.

\subsection{The Low-Degree Likelihood Ratio}

The so-called low-degree method for predicting the amount of computational power required for hypothesis testing tasks has seen fruitful development in the recent years, after its origination from studies on the sum-of-squares (SoS) hierarchy $\mathrm{BHK}^{+} 19, \mathrm{HS} 17, \mathrm{HKP}^{+} 17, \mathrm{Hop} 18$. This method has proven successful in understanding many classical statistical tasks, including community detection [HS17, Hop18, planted clique $\mathrm{BHK}^{+} 19, \mathrm{Hop} 18$, PCA and sparse PCA in spiked matrix models [BKW19, KWB19, DKWB19, and tensor PCA [HKP ${ }^{+} 17$, Hop18, KWB19]. Here we give a brief overview of this method; the reader may find more details in [HS17, Hop18] or in the survey article KWB19.

This method applies to hypothesis testing problems in which we aim to distinguish two sequences of hypotheses $\left\{\mathbb{P}_{N}\right\}$ and $\left\{\mathbb{Q}_{N}\right\}$, where $\mathbb{P}_{N}$ and $\mathbb{Q}_{N}$ are probability distributions on $\Omega_{N}=\mathbb{R}^{d(N)}$ with $d(N)=N^{O(1)}$. Usually $\mathbb{Q}_{N}$ is referred to as the "null" distribution (which contains pure noise), and $\mathbb{P}_{N}$ is referred to as the "planted" distribution (which contains a planted structure). We are interested in the problem of strongly distinguishing $\mathbb{P}$ from $\mathbb{Q}$ in a computationally-efficient manner, that is, we are interested in the minimal runtime needed for an algorithm that takes as input a sample from either $\mathbb{P}$ or $\mathbb{Q}$ and correctly identifies which of the two distributions it was drawn from, with both type I and type II errors $o(1)$ as $N \rightarrow \infty$.

The central idea of the low-degree method is to study a restricted class of algorithms, namely degree- $D$ multivariate polynomials $f: \Omega_{N} \rightarrow \mathbb{R}$, for a particular choice of $D=D(N)$ discussed later. We say that such a polynomial succeeds at distinguishing $\mathbb{P}$ from $\mathbb{Q}$ provided that

$$
\underset{Y \sim \mathbb{Q}_{N}}{\mathbb{E}}[f(Y)]=0, \underset{Y \sim \mathbb{P}_{N}}{\mathbb{E}}[f(Y)]=1, \underset{Y \sim \mathbb{Q}_{N}}{\operatorname{Var}}[f(Y)]=o(1), \underset{Y \sim \mathbb{P}_{N}}{\operatorname{Var}}[f(Y)]=o(1)
$$

as $N \rightarrow \infty$, in which case $\mathbb{P}$ and $\mathbb{Q}$ can be strongly distinguished by thresholding $f(Y)$. To prove failure of all degree- $D$ polynomials, we will be interested in computing the quantity

$$
\left\|L_{N}^{\leq D}\right\|:=\max _{f \in \mathbb{R}[Y] \leq D} \frac{\mathbb{E}_{Y \sim \mathbb{P}_{N}}[f(Y)]}{\sqrt{\mathbb{E}_{Y \sim \mathbb{Q}_{N}}\left[f(Y)^{2}\right]}}
$$

where $\mathbb{R}[Y]_{\leq D}$ is the space of polynomials $f: \Omega_{N} \rightarrow \mathbb{R}$ of degree (at most) $D$. Note that if $\left\|L_{N}^{\leq D}\right\|=$ $O(1)$ as $N \rightarrow \infty$ then no degree- $D$ polynomial can succeed in the sense of (1). The notation $\left\|L_{\bar{N}}^{\leq D}\right\|$ stems from the fact that this quantity is the $L^{2}\left(\mathbb{Q}_{N}\right)$-norm of the low-degree likelihood ratio $L_{N}^{\leq D}$, which is the orthogonal projection of the likelihood ratio $L_{N}=d \mathbb{P}_{N} / d \mathbb{Q}_{N}$ onto the subspace of degree- $D$ polynomials; see e.g. Hop18, KWB19]. 
Recent work has revealed that low-degree polynomials appear to be a good proxy for the inherent computational tractability of many high-dimensional testing problems. For many classical problems - including planted clique, sparse PCA, community detection, tensor PCA, and more - it has been shown that polynomials of degree $D=O(\log N)$ are as powerful as the best known polynomial-time algorithms. In other words, for all of these problems, in the parameter regime where a poly-time algorithm is known we have $\left\|L_{N}^{\leq O(\log N)}\right\| \rightarrow \infty$ whereas in the conjectured "hard" regime (where no poly-time algorithm is known) we have $\left\|L_{N}^{\leq \omega(\log N)}\right\|=O(1)$ HS17, $\mathrm{HKP}^{+}$17, Hop18, KWB19, DKWB19. This method even captures sharp computational thresholds such as the Kesten-Stigum bound in the stochastic block model [HS17, Hop18. One explanation for this phenomenon is that the best known poly-time algorithms for these types of problems typically take the form of spectral methods (i.e. thresholding the leading eigenvalue of some matrix built from the observed data), and any such spectral method can be implemented as an $O(\log N)$-degree polynomial via power iteration (under mild conditions); see Theorem 4.4 of [KWB19] for the precise sense in which boundedness of $\left\|L_{N}^{\leq D}\right\|$ imply failure of all spectral methods. Boundedness of $\left\|L_{N}^{\leq D}\right\|$ also implies failure of statistical query algorithms; see [BBH ${ }^{+} 20$. For larger degree $D \gg \log N$ it has also been observed that degree- $D$ polynomials are as powerful as the best known algorithms of runtime $N^{\tilde{\Theta}(D)}$ (which is the runtime needed to evaluate a degree- $D$ polynomial) in settings such as tensor PCA [KWB19] and sparse PCA [DKWB19] (both of which exhibit a smooth tradeoff between signal strength and subexponential runtime). The above ideas are summarized by the following informal low-degree conjecture based on [HS17, HKP ${ }^{+} 17$, Hop18.

Conjecture 1.6 (Informal). Let $t: \mathbb{N} \rightarrow \mathbb{N}$. For "natural" high-dimensional testing problems specified by $\mathbb{P}_{N}$ and $\mathbb{Q}_{N}$, if $\left\|L_{N}^{\leq D(N)}\right\|$ remains bounded as $N \rightarrow \infty$ whenever $D(N) \leq t(N)$. poly $\log (N)$, then there exists no sequence of functions $f_{N}: \Omega_{N} \rightarrow\{p, q\}$ with $f_{N}$ computable in time $N^{O(t(N))}$ that strongly distinguishes $\mathbb{P}_{N}$ and $\mathbb{Q}_{N}$, i.e., that satisfies

$$
\lim _{N \rightarrow \infty} \mathbb{Q}_{N}\left[f_{N}(Y)=q\right]=\lim _{N \rightarrow \infty} \mathbb{P}_{N}\left[f_{N}(Y)=p\right]=1 .
$$

This conjecture is informal because we have not attempted to specify the precise class of testing problems for which this is believed to hold. One formal variant of this conjecture is given by Conjecture 2.2.4 of Hop18, although it does not quite apply to the distributions that we consider in this paper. Still, we expect that Conjecture 1.6 holds for our particular choice of $\mathbb{P}$ and $\mathbb{Q}$ (defined in Section 1.3), as these distributions are very similar in spirit to many classical settings where the conjecture is known to coincide with widely believed computational thresholds (e.g. spiked matrix models [BKW19, KWB19] and sparse PCA [DKWB19]). We note that proving Conjecture 1.6 appears to be currently out of reach, as this would imply $P \neq N P$. Furthermore, current techniques are not able to prove computational hardness of average-case problems, even under the assumption $P \neq N P$. Instead, one should think of bounds on $\left\|L^{\leq D}\right\|$ as unconditional lower bounds against a restricted class of algorithms (namely low-degree polynomials). Conjecture 1.6 captures the idea that low-degree algorithms are optimal among all known algorithms for a large (and growing) number of problems of this flavor.

In this paper, we give bounds on $\left\|L^{\leq D}\right\|$ for our specific distributions $\mathbb{P}$ and $\mathbb{Q}$ defined in Section 1.3. Assuming Conjecture 1.6, this implies that runtime $N^{\tilde{\Omega}\left(s^{2} / M\right)}$ is required to distinguish them. Alternatively, the reader may choose to think of our lower bounds as unconditional lower bounds against low-degree algorithms. This suggests that beating the runtime $N^{\tilde{\Omega}\left(s^{2} / M\right)}$ would require a drastically new algorithmic technique which would likely lead to breakthroughs in other problems as well. 
Organization. The remainder of the paper is organized as follows. In Section 2, we state our lower bound for average-case RIP certification based on the low-degree likelihood ratio, and show that the lower bound is optimal, matching the upper bound due to [KZ14]. In Section 3, we give the proof for the lower bound.

Notation. Our standard asymptotic notation $O(\cdot), \Omega(\cdot), \Theta(\cdot)$ always pertains to the limit $N \rightarrow$ $\infty$. We use polylog $(N)$ to mean $(\log N)^{O(1)}$. We also use $\tilde{O}(B)$ to mean $O(B \cdot \operatorname{polylog}(N)), \tilde{\Omega}(B)$ to mean both $\Omega(B / \operatorname{polylog}(N))$, and $\tilde{\Theta}(B)$ to mean $\tilde{O}(B)$ and $\tilde{\Omega}(B)$. Also recall that $f(N)=o(g(N))$ (or equivalently $f(N) \ll g(N)$ ) means $f(N) / g(N) \rightarrow 0$ as $N \rightarrow \infty$ and $f(N)=\omega(g(N)$ ) (or equivalently $f(N) \gg g(N))$ means $f(N) / g(N) \rightarrow \infty$ as $N \rightarrow \infty$. We write $A \lesssim B$ to mean $A \leq C B$ for an absolute constant $C$, and $A \gtrsim B$ to mean $A \geq C B$ for an absolute constant $C$. We say that an event occurs with high probability if it occurs with probability $1-o(1)$ as $N \rightarrow \infty$.

\section{Main Results}

We follow the proof sketch outlined in Proposition 1.5. Adopting the framework of Proposition 1.5. we consider $\delta \in(0,1)$ held fixed as $N \rightarrow \infty$, with $M=M(N)$ and $s=s(N)$ scaling with $N$ such that $M \leq N, M \rightarrow \infty$ and $s \rightarrow \infty$.

Lemma 2.1. Consider the setting of Proposition 1.5. Under $\mathbb{P}$,

$$
\operatorname{Pr}\left[\frac{1}{\sqrt{M}} A \text { is }(s, \delta)-\mathrm{RIP}\right] \leq \exp \left(-\frac{\delta^{2} M}{12}\right)+2 \exp \left(-\frac{(1-\delta)^{2} s}{24}\right)=o(1)
$$

We defer the proof of Lemma 2.1 to Section 3. The following well-known result tells us that the random ensemble is RIP with high probability under $\mathbb{Q}$.

Theorem 2.2 (See [WBP16], Proposition 1; also [CT05, BDDW08, FR13]). In the setting of Proposition 1.5 , under $\mathbb{Q}$,

$$
\operatorname{Pr}\left[\frac{1}{\sqrt{M}} A \text { is not }(s, \delta)-\mathrm{RIP}\right] \leq 2 \exp \left[s \log \left(\frac{9 e N}{s}\right)-\frac{\delta^{2} M}{256}\right]
$$

which is o(1) provided $s \lesssim M / \log N$.

Based on Conjecture 1.6, we are left to show the boundedness of the low-degree likelihood ratio between $\mathbb{P}$ and $\mathbb{Q}$, which gives evidence for hardness of the certification task. This is demonstrated in the following theorem.

Theorem 2.3. In the setting of Proposition 1.5, suppose $M=M(N), s=s(N)$, and $D=D(N)$ satisfy $s \leq M$ and $D=o\left(s^{2} / M\right)$. Then the low-degree likelihood ratio $\left\|L^{\leq D}\right\|$ of $\mathbb{P}$ against $\mathbb{Q}$ remains bounded as $N \rightarrow \infty$.

The proof of Theorem 2.3 is deferred to Section 3. The condition $s \leq M$ is not restrictive because we are interested in the regime $s \lesssim M / \log N$ so that a random matrix drawn from $\mathbb{Q}$ is RIP. (Our proof actually still works without the assumption $s \leq M$, provided we add the additional assumption $D=o(M)$.) Theorem 2.3 tells us that for a polynomial to distinguish $\mathbb{P}$ from $\mathbb{Q}$, it must have degree at least $\Omega\left(s^{2} / M\right)$. As discussed in Section 1.4, this suggests that runtime $N^{\tilde{\Omega}\left(s^{2} / M\right)}$ is required to strongly distinguish $\mathbb{P}$ from $\mathbb{Q}$ (at least with current techniques).

Together, the results in this section verify items (1)-(3) in Proposition 1.5 and thus constitute evidence that RIP certification requires time $N^{\tilde{\Omega}\left(s^{2} / M\right)}$. 
Remark 2.4 (Relation to planted sparse vector problem). Note that the orthogonal complement of the row-span of our negatively-spiked Wishart matrix is a subspace of dimension $N-M$ that (approximately) contains a sparse vector. Thus Theorem 2.3 suggests that when $s \geq \sqrt{N} \cdot \operatorname{polylog}(N)$, no polynomial-time algorithm can recover a planted $s$-sparse vector in a random subspace of dimension $\Theta(N)$ in $\mathbb{R}^{N}$. This matches the upper bound of the $l^{1} / l^{\infty}$ relaxation in [SWW12, DH14]. Another line of work on finding a sparse vector in a subspace [BKS14, QSW14, HSSS16] considers the setting where $s=O(N)$ and the subspace has dimension o $(N)$, but our results do not apply to this regime.

For convenience to the reader, we also present below the lazy algorithm proposed in [KZ14, which gives the matching upper bound. In the following context, for $S \subseteq[N]$ we denote by $\boldsymbol{P}_{S}:=\sum_{i \in S} e_{i} e_{i}^{\top}$ the projector that zeros out all but the entries indexed by $S$ of a vector.

Proposition 2.5. For a matrix $X \in \mathbb{R}^{M \times N}$ and $s \in \mathbb{N}_{+}$, for any $\delta \in(0,1)$, X satisfies the $(s, \delta)$-RIP if and only if

$$
B_{s}(X):=\max _{\substack{S \subseteq[N] \\|S|=s}}\left\|\boldsymbol{P}_{S}\left(X^{\top} X-I_{N}\right) \boldsymbol{P}_{S}\right\| \leq \delta .
$$

Proposition 2.5 follows directly from the definition of RIP. A naïve way to certify RIP is to compute $B_{s}(X)$ by enumerating all $S \subseteq[N]$ with $|S|=s$, which would take time $N^{\Theta(s)}$. Instead, the following algorithm calculates $B_{r}(X)$ for $r=\tilde{\Theta}\left(s^{2} / M\right)$, thereby reducing the total runtime to $N^{\tilde{\Theta}\left(s^{2} / M\right)}$.

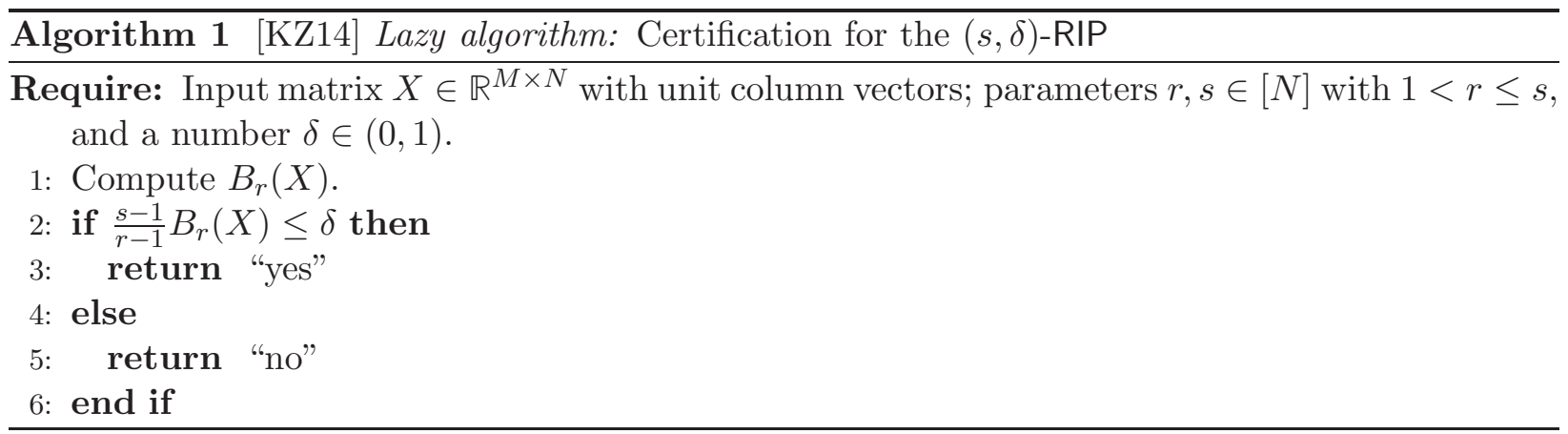

It is proved in KZ14 that, with a choice $r=\tilde{\Theta}\left(s^{2} /\left(\delta^{2} M\right)\right)$, Algorithm 1 certifies the $(s, \delta)$-RIP for $X=\frac{1}{\sqrt{M}} A$ with high probability, where $A$ is an $M \times N$ matrix with i.i.d. symmetric $( \pm 1)$ Bernoulli random variables as its entries. We remark that with minor changes the same procedure works for $A$ with i.i.d. standard Gaussian random variables, and possibly also for many other ensembles. Based on our lower bound and the effectiveness of Algorithm 1, we conclude that the task of $(s, \delta)$ RIP certification requires time precisely $N^{\tilde{\Theta}\left(s^{2} / M\right)}$. However, our lower bound (Theorem 2.3) does not seem to easily generalize to other ensembles, since we have used gaussianity in a crucial way.

Remark 2.6 (Relation to sparse PCA). We remark that the RIP certification problem bears resemblance to sparse PCA, which is the positively-spiked $(\beta>0)$ case of the spiked Wishart model with a sparse spike prior. In [DKWB19], the authors investigated the precise runtime required to solve sparse PCA. This includes an algorithm that improves over the runtime of naïve exhaustive search by enumerating subsets of a particular size smaller than the true sparsity (similar to the lazy algorithm above and to the sparse PCA algorithm of [HSV19]), as well as a matching lower bound based on the low-degree likelihood ratio. 


\section{Proof for the Low-Degree Likelihood Ratio Bound}

In this section we prove Lemma 2.1 and Theorem 2.3. We start with introducing the following two Chernoff-type bounds for Bernoulli and $\chi^{2}$ sums.

Lemma 3.1. Suppose $x$ is taken from the sparse Rademacher prior $\mathcal{X}_{\rho}^{N}$ per Definition 1.4 . For any $\mu \in(0,1]$, we have

$$
\operatorname{Pr}\left[\|x\|^{2}>1+\mu\right] \leq \exp \left(-\frac{\mu^{2} \rho N}{3}\right), \quad \operatorname{Pr}\left[\|x\|^{2}<1-\mu\right] \leq \exp \left(-\frac{\mu^{2} \rho N}{2}\right)
$$

and therefore

$$
\operatorname{Pr}\left[1-\mu \leq\|x\|^{2} \leq 1+\mu\right] \geq 1-2 \exp \left(-\frac{\mu^{2} \rho N}{3}\right)
$$

Proof. Note that

$$
\operatorname{Pr}\left[\|x\|^{2}>1+\mu\right]=\operatorname{Pr}\left[\|x\|_{0}>(1+\mu) \rho N\right], \quad \operatorname{Pr}\left[\|x\|^{2}<1-\mu\right]=\operatorname{Pr}\left[\|x\|_{0}<(1-\mu) \rho N\right]
$$

where $\|x\|_{0}$ is the sum of $N$ independent $\operatorname{Bernoulli}(\rho)$ random variables, and $\mathbb{E}\|x\|_{0}=\rho N$. Therefore (5) and (6) follow from the multiplicative Chernoff bound in AV79.

Lemma 3.2 (Chernoff bound for $\chi^{2}$ distribution). For all $\delta \in(0,1)$,

$$
\operatorname{Pr}\left[\frac{1}{M} \chi_{M}^{2} \geq 1+\delta\right] \leq \exp \left(-\frac{\delta^{2} M}{12}\right)
$$

Proof. By the classical Chernoff bound (see, for example [LM00]),

$$
\frac{1}{M} \operatorname{Pr}\left[\chi_{M}^{2} \geq(1+\delta) M\right] \leq \frac{1}{2}(-\delta+\log (1+\delta)) .
$$

Now the lemma follows immediately from the observation that for $\delta \in(0,1)$,

$$
\frac{1}{2}(-\delta+\log (1+\delta)) \leq-\frac{\delta^{2}}{12}
$$

Proof of Lemma 2.1. Note that

$$
\left\|\frac{1}{\sqrt{M}} A x\right\|^{2}=\frac{1}{M} \sum_{i=1}^{M}\left(u_{i}^{\top} x\right)^{2} \sim \frac{1}{M}\left(\|x\|^{2}-(1-\epsilon)\|x\|^{4}\right) \chi_{M}^{2} .
$$

For $x$ taken from $\mathcal{X}_{N}^{\rho}$ satisfying $1-\epsilon \leq\|x\|^{2} \leq 1+\epsilon$, since $-(1-\epsilon)\|x\|^{2} \geq-\left(1-\epsilon^{2}\right)>-1$, under $\mathbb{P}$ each row of the observation $A$ is taken from $\mathcal{N}\left(0, I-(1-\epsilon) x x^{\top}\right)$. Furthermore, we deduce from $\|x\|^{2} \leq 1+\epsilon$ that $\|x\|_{0} \leq(1+\epsilon) \rho_{N} N \leq s$. Now Lemma 3.2 gives

$$
\begin{aligned}
\operatorname{Pr}\left[\left\|\frac{1}{\sqrt{M}} A x\right\|^{2} \geq(1-\delta)\|x\|^{2}\right] & =\operatorname{Pr}\left[\frac{1}{M} \chi_{M}^{2} \geq \frac{1-\delta}{1-(1-\epsilon)\|x\|^{2}}\right] \\
& \leq \operatorname{Pr}\left[\frac{1}{M} \chi_{M}^{2} \geq 1+\delta\right] \\
& \leq \exp \left(-\frac{\delta^{2} M}{12}\right) .
\end{aligned}
$$


The first inequality used the fact that

$$
\frac{1-\delta}{1-(1-\epsilon)\|x\|^{2}} \geq \frac{1-\delta}{1-(1-\epsilon)^{2}} \geq \frac{1-\delta}{2 \epsilon}=1+\delta
$$

Therefore we know from Lemma 3.1 that

$$
\begin{aligned}
& \operatorname{Pr}\left[\frac{1}{\sqrt{M}} A \text { is }(s, \delta)-\mathrm{RIP}\right] \\
\leq & \operatorname{Pr}\left[1-\epsilon \leq\|x\|^{2} \leq 1+\epsilon,\left\|\frac{1}{\sqrt{M}} A x\right\|^{2} \geq(1-\delta)\|x\|^{2}\right]+\operatorname{Pr}\left[\|x\|^{2}<1-\epsilon\right]+\operatorname{Pr}\left[\|x\|^{2}>1+\epsilon\right] \\
\leq & \exp \left(-\frac{\delta^{2} M}{12}\right)+2 \exp \left(-\frac{(1-\delta)^{2} s}{24}\right)
\end{aligned}
$$

the rightmost sum being $o(1)$ given $M \rightarrow \infty$ and $s \rightarrow \infty$ as $N \rightarrow \infty$.

Proof of Theorem 2.3. Let $L_{N, M, \beta, \mathcal{X}}^{\leq D}$ denote the degree- $D$ likelihood ratio for the spiked Wishart model (see Definition 1.3) with parameters $N, M, \beta$ and spike prior $\mathcal{X}$. [BKW19] gives the formula

$$
\begin{aligned}
\left\|L_{N, M, \beta, \mathcal{X}}^{\leq D}\right\|_{2}^{2} & =\underset{v^{(1)}, v^{(2)} \sim \mathcal{X}_{N}}{\mathbb{E}}\left[\varphi_{M,\lfloor D / 2\rfloor}\left(\frac{\beta^{2}\left\langle v^{(1)}, v^{(2)}\right\rangle^{2}}{4}\right)\right] \\
& =\underset{v^{(1)}, v^{(2)} \sim \mathcal{X}_{N}}{\mathbb{E}} \sum_{d=0}^{\lfloor D / 2\rfloor}\left(\sum_{\substack{d_{1}, \ldots, d_{M} \\
\sum d_{i}=d}}^{M} \prod_{i=1}^{M}\left(\begin{array}{c}
2 d_{i} \\
d_{i}
\end{array}\right)\right)\left(\frac{\beta^{2}\left\langle v^{(1)}, v^{(2)}\right\rangle^{2}}{4}\right)^{d},
\end{aligned}
$$

where $v^{(1)}, v^{(2)}$ are drawn independently from $\mathcal{X}_{N}$. Here $\varphi_{N, k}(x)$ is the Taylor series of $\varphi_{N}$ around $x=0$ truncated to degree $k$, i.e.

$$
\begin{aligned}
\varphi_{M}(x) & :=(1-4 x)^{-M / 2} \\
\varphi_{M, k}(x) & :=\sum_{d=0}^{k} x^{d} \frac{1}{d !} \prod_{a=0}^{d-1}(2 M+4 a)
\end{aligned}
$$

by the the generalized binomial theorem, where the coefficient of $x^{d}$ may be written in terms of a generalized binomial coefficient as $(-4)^{d}\left(\begin{array}{c}-M / 2 \\ d\end{array}\right) 11$ Note that under the setting of Problem 1.5, we are essentially dealing with $\mathcal{X}_{N}$ a truncated version of the sparse Rademacher prior $\mathcal{X}_{N}^{\rho_{N}}: x \sim \mathcal{X}_{N}$ is taken as following.

(1) Draw $x \sim \mathcal{X}_{N}^{\rho_{N}}$.

(2) If $-(1-\epsilon)\|x\|^{2}<-1$, then set $x=0$.

Therefore for any non-negative integer $d$ it holds that

$$
\underset{v^{(1)}, v^{(2)} \sim \mathcal{X}_{N}}{\mathbb{E}}\left\langle v^{(1)}, v^{(2)}\right\rangle^{2 d} \leq \underset{v^{(1)}, v^{(2)} \sim \mathcal{X}_{N}^{\rho_{N}}}{\mathbb{E}}\left\langle v^{(1)}, v^{(2)}\right\rangle^{2 d} .
$$

\footnotetext{
${ }^{1}$ We note that this expansion of the power series, which allows a simplified analysis, was not noticed in BKW19.
} 
For any $d \leq D$, the power series coefficients above are bounded by

$$
0 \leq \frac{1}{d !} \prod_{a=0}^{d-1}(2 M+4 a) \leq \frac{(2 M+4 D)^{d}}{d !}
$$

For independent choices of $v^{(1)}, v^{(2)} \sim \mathcal{X}_{N}^{\rho_{N}}$, denote $S^{(1)}$ and $S^{(2)}$ their respective support. Observe that

$$
\left\langle v^{(1)}, v^{(2)}\right\rangle \mid S^{(1)}, S^{(2)} \stackrel{d}{=} \frac{1}{\rho N} \sum_{i \in S^{(1)} \cap S^{(2)}} R_{i}
$$

where $R_{i}$ are i.i.d. Rademacher random variables. Following Section 4.2 of [LWB20], we get

$$
\begin{aligned}
v_{v^{(1)}, v^{(2)} \sim \mathcal{X}_{N}^{\rho_{N}}}^{\mathbb{E}}\left\langle v^{(1)}, v^{(2)}\right\rangle^{2 d} & =\frac{1}{(\rho N)^{2 d}} \underset{S^{(1)}, S^{(2)}}{\mathbb{E}}\left[\mathbb{E}\left(\sum_{i \in S^{(1)} \cap S^{(2)}} R_{i}\right)^{2 d} \mid S^{(1)}, S^{(2)}\right] \\
& \leq \frac{1}{(\rho N)^{2 d}}(2 d-1) !{\underset{S}{(1)}, S^{(2)}}_{\mid \mathbb{E}}\left|S^{(1)} \cap S^{(2)}\right|^{d} \\
& =\frac{1}{(\rho N)^{2 d}}(2 d-1) ! ! \cdot \mathbb{E}\left(\sum_{i=1}^{N} B_{i}\right)^{d} \\
& \leq \frac{1}{(\rho N)^{2 d}}(2 d-1) ! !\left[\rho^{2} N+2^{1 / d}\left(d^{d / 2} 4^{d-1}\left(\rho^{2} N\right)^{d / 2}+\left(\frac{4}{3} d\right)^{d}\right)^{1 / d}\right]^{d} \\
& \leq \frac{1}{(\rho N)^{2 d}}(2 d-1) ! !\left(\rho^{2} N+4 \rho \sqrt{d N}+3 d\right)^{d}
\end{aligned}
$$

where, in (11), $B_{i}=\mathbf{1}\left\{i \in S^{(1)} \cap S^{(2)}\right\}$ are i.i.d. Bernoulli random variables with success probability $\rho^{2}$. Overall, we obtain that

$$
\begin{aligned}
\left\|L_{N, M, \beta, \mathcal{X}}^{\leq D}\right\|_{2}^{2} & \lesssim \sum_{d=0}^{\lfloor D / 2\rfloor}\left(\frac{\beta^{2}(M+2 D)}{\rho^{2} N^{2}}\right)^{d}\left(\rho^{2} N+4 \rho \sqrt{d N}+3 d\right)^{d} \\
& \leq \sum_{d=0}^{\lfloor D / 2\rfloor}\left(\frac{\beta^{2}(M+2 D)}{\rho^{2} N^{2}}\right)^{d}\left(\rho^{2} N+4 \rho \sqrt{D N / 2}+\frac{3 D}{2}\right)^{d} \\
& \leq \sum_{d=0}^{\lfloor D / 2\rfloor}\left(\beta^{2}\left(\frac{M+2 D}{N}+\frac{4 \sqrt{2 D}(M+2 D)}{s \sqrt{N}}+\frac{6 D(M+2 D)}{s^{2}}\right)\right)^{d} \\
& =\sum_{d=0}^{\lfloor D / 2\rfloor}\left((1+o(1))(1-\epsilon)^{2}\left(\frac{M}{N}+4 \sqrt{2} \sqrt{\frac{D M}{s^{2}}} \sqrt{\frac{M}{N}}+6 \frac{D M}{s^{2}}\right)\right)^{d} \\
& =O(1)
\end{aligned}
$$

where we have used $\beta=-(1-\epsilon)$ and $M \leq N$ along with the assumptions from Theorem 2.3: $s \leq M$ and $D=o\left(s^{2} / M\right)$, which together imply $D=o(M)$.

\section{Acknowledgments}

We thank Cheng Mao for helpful comments on an earlier version. 


\section{References}

[AHSC09] Lorne Applebaum, Stephen D Howard, Stephen Searle, and Robert Calderbank. Chirp sensing codes: Deterministic compressed sensing measurements for fast recovery. Applied and Computational Harmonic Analysis, 26(2):283-290, 2009.

[AV79] Dana Angluin and Leslie G Valiant. Fast probabilistic algorithms for hamiltonian circuits and matchings. Journal of Computer and system Sciences, 18(2):155-193, 1979 .

$\left[\mathrm{BBH}^{+} 20\right]$ Matthew Brennan, Guy Bresler, Samuel B Hopkins, Jerry Li, and Tselil Schramm. Statistical query algorithms and low-degree tests are almost equivalent. arXiv preprint arXiv:2009.06107, 2020.

[BDDW08] Richard Baraniuk, Mark Davenport, Ronald DeVore, and Michael Wakin. A simple proof of the restricted isometry property for random matrices. Constructive Approximation, 28(3):253-263, 2008.

$\left[\mathrm{BDF}^{+} 11\right]$ Jean Bourgain, Stephen Dilworth, Kevin Ford, Sergei Konyagin, and Denka Kutzarova. Explicit constructions of RIP matrices and related problems. Duke Mathematical Journal, 159(1):145-185, 2011.

[BDMS13] Afonso S Bandeira, Edgar Dobriban, Dustin G Mixon, and William F Sawin. Certifying the restricted isometry property is hard. IEEE transactions on information theory, 59(6):3448-3450, 2013.

[BFMM16] Afonso S Bandeira, Matthew Fickus, Dustin G Mixon, and Joel Moreira. Derandomizing restricted isometries via the legendre symbol. Constructive Approximation, 43(3):409-424, 2016.

[BFMW13] Afonso S Bandeira, Matthew Fickus, Dustin G Mixon, and Percy Wong. The road to deterministic matrices with the restricted isometry property. Journal of Fourier Analysis and Applications, 19(6):1123-1149, 2013.

$\left[\mathrm{BHK}^{+} 19\right]$ Boaz Barak, Samuel Hopkins, Jonathan Kelner, Pravesh K Kothari, Ankur Moitra, and Aaron Potechin. A nearly tight sum-of-squares lower bound for the planted clique problem. SIAM Journal on Computing, 48(2):687-735, 2019.

[BKS14] Boaz Barak, Jonathan A Kelner, and David Steurer. Rounding sum-of-squares relaxations. In Proceedings of the forty-sixth annual ACM symposium on Theory of computing, pages 31-40, 2014.

[BKW19] Afonso S Bandeira, Dmitriy Kunisky, and Alexander S Wein. Computational hardness of certifying bounds on constrained PCA problems. arXiv preprint arXiv:1902.07324, 2019 .

[BMM17] Afonso S Bandeira, Dustin G Mixon, and Joel Moreira. A conditional construction of restricted isometries. International Mathematics Research Notices, 2017(2):372-381, 2017.

[Can08] Emmanuel J Candès. The restricted isometry property and its implications for compressed sensing. Comptes rendus mathematique, 346(9-10):589-592, 2008. 
[CT05] Emmanuel J Candès and Terence Tao. Decoding by linear programming. IEEE Transactions on information theory, 51(12):4203, 2005.

[CZ13a] T Tony Cai and Anru Zhang. Sharp RIP bound for sparse signal and low-rank matrix recovery. Applied and Computational Harmonic Analysis, 35(1):74-93, 2013.

[CZ13b] T Tony Cai and Anru Zhang. Sparse representation of a polytope and recovery of sparse signals and low-rank matrices. IEEE Transactions on Information Theory, 60(1):122$132,2013$.

[DeV07] Ronald A DeVore. Deterministic constructions of compressed sensing matrices. Journal of complexity, 23(4):918-925, 2007.

[DH14] Laurent Demanet and Paul Hand. Scaling law for recovering the sparsest element in a subspace. Information and Inference: A Journal of the IMA, 3(4):295-309, 2014.

[DKWB19] Yunzi Ding, Dmitriy Kunisky, Alexander S Wein, and Afonso S Bandeira. Subexponential-time algorithms for sparse PCA. arXiv preprint arXiv:190\%.11635, 2019 .

[Don06] David L Donoho. Compressed sensing. IEEE Transactions on information theory, 52(4):1289-1306, 2006.

[FMT12] Matthew Fickus, Dustin G Mixon, and Janet C Tremain. Steiner equiangular tight frames. Linear algebra and its applications, 436(5):1014-1027, 2012.

[FR13] Simon Foucart and Holger Rauhut. A Mathematical Introduction to Compressive Sensing. Springer, 2013.

[Gam18] David Gamarnik. Explicit construction of RIP matrices is Ramsey-hard. arXiv preprint arXiv:1805.11238, 2018.

$\left[\mathrm{HKP}^{+} 17\right]$ Samuel B Hopkins, Pravesh K Kothari, Aaron Potechin, Prasad Raghavendra, Tselil Schramm, and David Steurer. The power of sum-of-squares for detecting hidden structures. In 2017 IEEE 58th Annual Symposium on Foundations of Computer Science (FOCS), pages 720-731. IEEE, 2017.

[Hop18] Samuel B Hopkins. Statistical Inference and the Sum of Squares Method. PhD thesis, Cornell University, 2018.

[HS17] Samuel B Hopkins and David Steurer. Bayesian estimation from few samples: community detection and related problems. arXiv preprint arXiv:1710.00264, 2017.

[HSSS16] Samuel B Hopkins, Tselil Schramm, Jonathan Shi, and David Steurer. Fast spectral algorithms from sum-of-squares proofs: tensor decomposition and planted sparse vectors. In Proceedings of the forty-eighth annual ACM symposium on Theory of Computing, pages 178-191, 2016.

[HSV19] Guy Holtzman, Adam Soffer, and Dan Vilenchik. A greedy anytime algorithm for sparse PCA. arXiv preprint arXiv:1910.06846, 2019.

[KWB19] Dmitriy Kunisky, Alexander S Wein, and Afonso S Bandeira. Notes on computational hardness of hypothesis testing: Predictions using the low-degree likelihood ratio. arXiv preprint arXiv:190\%.11636, 2019. 
[KZ14] Pascal Koiran and Anastasios Zouzias. Hidden cliques and the certification of the restricted isometry property. IEEE transactions on information theory, 60(8):49995006, 2014.

[LM00] Beatrice Laurent and Pascal Massart. Adaptive estimation of a quadratic functional by model selection. Annals of Statistics, pages 1302-1338, 2000.

[LWB20] Matthias Löffler, Alexander S Wein, and Afonso S Bandeira. Computationally efficient sparse clustering. arXiv preprint arXiv:2005.10817, 2020.

[Mix15] Dustin G Mixon. Explicit matrices with the restricted isometry property: Breaking the square-root bottleneck. In Compressed sensing and its applications, pages 389-417. Springer, 2015.

[NW14] Abhiram Natarajan and Yi Wu. Computational complexity of certifying restricted isometry property. arXiv preprint arXiv:1406.5791, 2014.

[QSW14] Qing Qu, Ju Sun, and John Wright. Finding a sparse vector in a subspace: Linear sparsity using alternating directions. In Advances in Neural Information Processing Systems, pages 3401-3409, 2014.

[SWW12] Daniel A Spielman, Huan Wang, and John Wright. Exact recovery of sparsely-used dictionaries. In Conference on Learning Theory, pages 37-1, 2012.

[TP13] Andreas M Tillmann and Marc E Pfetsch. The computational complexity of the restricted isometry property, the nullspace property, and related concepts in compressed sensing. IEEE Transactions on Information Theory, 60(2):1248-1259, 2013.

[WBP16] Tengyao Wang, Quentin Berthet, and Yaniv Plan. Average-case hardness of RIP certification. In Advances in Neural Information Processing Systems, pages 3819-3827, 2016.

[Wee17] Jonathan Weed. Approximately certifying the restricted isometry property is hard. IEEE Transactions on Information Theory, 64(8):5488-5497, 2017. 